\title{
Influence of respiratory movement during regional nodal irradiation after mastectomy for breast cancer: an experimental study
}

Hosang Jeon

Pusan National University Yangsan Hospital

\section{Yongkan Ki}

Pusan National University School of Medicine

Dong Woon Kim

Pusan National University Yangsan Hospital

\section{Wontaek Kim}

Pusan National University School of Medicine

\section{Jiho Nam}

Pusan National University Hospital

\section{Donghyun Kim}

Pusan National University Hospital

\section{Dahl Park}

Pusan National University Hospital

Ji Hyeon Joo ( $\nabla$ hi_juji@daum.net)

Pusan National University Yangsan Hospital https://orcid.org/0000-0001-9275-3197

\section{Research}

Keywords: Breast cancer, Internal mammary lymph nodes, Radiation therapy, Volumetric modulated arc therapy

Posted Date: May 13th, 2020

DOI: https://doi.org/10.21203/rs.3.rs-27983/v1

License: (c) (i) This work is licensed under a Creative Commons Attribution 4.0 International License. Read Full License 


\section{Abstract}

Background This study aimed to evaluate the dosimetric consequences of respiratory movement in postmastectomy radiation therapy (PMRT) including internal mammary nodes (IMNs) between volumetric modulated arc therapy (VMAT) and three-dimensional conformal radiation therapy (3D-CRT).

Methods An anthropomorphic phantom was used to mimic the chest anatomy of a patient who had undergone mastectomy. Two types of absorbed dose measurements were adopted; a radiochromic film was inserted into a gap between phantom slices at the level of the second IMN and three glass rod dosimeters were placed at the first IMN (IM), chest wall (CW), and left anterior descending (LAD) artery. Respiratory movements with amplitudes of $5 \mathrm{~mm}$ (R05) and $10 \mathrm{~mm}$ (R10) were simulated using a dynamic platform combined with a motorized jack. To evaluate dose errors caused by respiratory movement, the measured data in the presence and absence of respiratory movement were compared.

Results At IM, dose errors were - 2.8\% (R05) and - 6.2\% (R10) for 3D-CRT and - 4.9\% (R05) and - 8.5\% (R10) for VMAT. The dose errors in CW were - $0.5 \%$ (R05) and - $6.0 \%$ (R10) for 3D-CRT and - 1.9\% (R05) and $-5.3 \%$ (R10) for VMAT. The LAD doses showed very small absolute values. According to film measurements, dose errors of IMN were similar between 3D-CRT and VMAT, but the dose error of the lung was higher in 3D-CRT. The gamma pass rates of VMAT ( $97 \%$ at R05; $88 \%$ at R10) were higher than those of 3D-CRT (74\% at R05; $59 \%$ at R10).

Conclusions If the patient maintained shallow to normal breathing, PMRT including IMNs could be implemented with acceptable accuracy. In particular, it was possible to maintain the advantages of VMAT, which enabled high-target coverage and normal organ protection.

\section{Background}

Radiation therapy (RT) after mastectomy plays an important role in the management of locally advanced breast cancer. By reducing the risk of recurrence, postmastectomy RT (PMRT) offers an incremental improvement in breast cancer mortality and overall survival (1-3). Moreover, since recent evidence supported that PMRT in stage II breast cancer patients yielded improved locoregional control and 20-year breast cancer survival, a significant increase in the administration of PMRT has been reported $(1,4)$. PMRT in these studies was delivered to the chest wall and lymphatic drainage regions, including the axilla, supraclavicular fossa, and internal mammary nodes (IMNs). However, irradiation of IMN increases radiation exposure to the heart, which is directly related to the development of ischemic heart disease (5, 6). One of the treatment planning techniques to reduce cardiac dose is volumetric modulated arc therapy (VMAT). The VMAT involves continuous gantry rotation during the delivery of typically coplanar beams with variable shapes and intensities in a single gantry rotation $(7,8)$. Compared to conventional intensitymodulated RT (IMRT), the VMAT technique has been shown to significantly improve sparing of the heart and lungs while generating conformal and homogeneous dose distributions to the target in breast cancer patients (9). 
Although it has been proven to be excellent in dosimetry, monitor unit, and treatment time, whether VMAT will be implemented well in vivo as well needs further investigation. Obtaining adequate dose coverage of the IMN is challenging owing to the continuous target movement in respiration. Moreover, the IMN is surrounded by complex anatomical structures such as muscles, bones, and lungs in proximity. These could cause significant errors in the treatment dose delivery.

This study aimed to evaluate the dosimetric consequence of respiratory movements by simulating the PMRT in the presence of respiratory movement. For this purpose, an anthropomorphic phantom and a dynamic platform with a motorized jack were used. We focused on the comparison between conventional three-dimensional conformal RT (3D-CRT) and VMAT.

\section{Methods}

Target definition and treatment planning

An anthropomorphic phantom (Alderson Radiation Therapy Phantom, RSD, CA) was used to mimic the chest anatomy of a patient who had undergone mastectomy. The phantom was scanned with a $5 \mathrm{~mm}$ slice thickness on computed tomography (LightSpeed RT 16, GE, USA). The planning target volume (PTV) for the chest wall was delineated within the following six anatomical borders: (1) cranial: the caudal border of the clavicle head; (2) Caudal: presumed inframammary fold; (3) anterior: $3 \mathrm{~mm}$ under the skin surface; (4) posterior: rib-pleural interface; (5) lateral: mid-axillary line; and (6) medial sternal-rib junction. The PTV for the IMN was also delineated within the following borders: (1) cranial: superior aspect of the medial first rib and (2) caudal: cranial aspect of the fourth rib. A lateral thickness of $2 \mathrm{~cm}$ was applied to the clinical target volume (CTV) for the IMN to encompass the IMN vessels.

We made two radiation treatment plans (RTP) using 3D-CRT and VMAT. As a comparable conventional forward-planning technique, the 3D-CRT plan comprised two opposite tangential treatment fields that consisted of two segments each using an RTP platform (Eclipse ${ }^{\mathrm{TM}}$, Varian Medical Systems, CA). The treatment dose was delivered using a linear accelerator (Clinac iX, Varian Medical Systems, CA). Meanwhile, the VMAT was designed and subsequently optimized to minimize the defined cost functions using a dedicated RTP platform (Monaco ${ }^{\mathrm{TM}}$, Elekta, Sweden). The treatment dose was delivered using a linear accelerator (Versa HD, Elekta, Sweden). The detailed plan parameters are summarized in Table 1, and the field geometries are described in Fig. 1. 
Table 1

Comparison of radiation treatment planning conditions

\begin{tabular}{|lll|}
\hline Technique & 3D-CRT & VMAT \\
\hline RTP platform & Eclipse (v8.6.23) & Monaco (v5.11.02) \\
\hline Calculation algorithm & AAA (v8.9.17) & XVMC \\
\hline Prescription & $50 \mathrm{~Gy}(25 \mathrm{fx})$ at 95\% of CTV \\
\hline X-ray energy & $15 \mathrm{MV}$ & $6 \mathrm{MV}$ \\
\hline Gantry angle (MU) & $128(106), 308(113)$ & Half arc (844) \\
\hline Machine & Clinac iX, Varian & Versa HD, Elekta \\
\hline
\end{tabular}

Experimental setup

A two-dimensional dynamic platform was fabricated by combining a motorized jack system (in-house) and a commercial one-dimensional moving system (Model 008PL, CIRS, USA) to simulate the anteriorposterior and superior-inferior respiratory movements, respectively. The lateral movement was ignored because it was relatively small. Two-dimensional respiratory movement was successfully realized by synchronizing the time periods between the two systems. The time period was fixed at $3 \mathrm{~s}$. The overall experimental setup is presented in Fig. 2. To evaluate the influence of the magnitude of respiratory motion on the perturbation of dose distribution, the planned dose was repeatedly delivered three times while the anthropomorphic phantom was reciprocating at fixed intervals of $0 \mathrm{~mm}$ (R00), $5 \mathrm{~mm}$ (R05), and $10 \mathrm{~mm}$ (R10) for 3D-CRT and VMAT. Using two orthogonal kV imaging fields, the phantom was precisely located at the central position prior to the reciprocating motion.

\section{Measurements}

Two types of absorbed dose measurements were adopted to evaluate the delivered dose values in this study. First, a radiochromic film patch (EBT3, Ashland, USA) was inserted into a gap between two consecutive phantom slices to measure the absorbed dose distribution covering the second internal mammary chain and the neighboring chest wall (CW) area. The film measurement results were visualized and analyzed using the gamma evaluation method with the criteria of $3 \%$ dose and $3 \mathrm{~mm}$ distance (10). The minimum dose threshold for gamma evaluation was $10 \%$. In addition, we set two square regions of interest at the IMN ( $\left.D_{\text {IMN }}\right)$ in the PTV and lung ( $\left.D_{\text {lung }}\right)$ near the PTV, as shown in Fig. 4a and Fig. 4b, respectively. The average doses of $D_{I M N}$ and $D_{\text {lung }}$ were acquired for the evaluation of target and organ at risk (OAR) doses.

Second, three glass rod dosimeters (GRDs) (GD-302M, Asahi Techno glass, Japan) were placed to measure the delivered point doses-one at the first internal mammary chain (IM), one at the CW, and one at the left anterior descending artery (LAD). Both the IM and CW points were located inside the PTV, and the $\mathrm{CW}$ was also laid at the center of the presumed pectoralis muscle above the ribs. The LAD was 
located at the apex of the left ventricle. To avoid the measurement error caused by the inter-element deviation, the same GRD element was repeatedly used for the same position in the phantom during three irradiation rounds with three different reciprocating intervals. All GRD elements were scanned five times, and the averaged values were adopted.

Because this study focused on the impact of respiratory motion on the absorbed dose error, both film and GRD measurements under R05 and R10 conditions were compared with those under the R00 condition.

\section{Results}

All measured data in IM, CW, and LAD using GRDs, and $D_{I M N}$ and $D_{\text {lung }}$ using films are tabulated in Table 2, and the GRD readings are plotted in Fig. 3. The film measurements and gamma analysis are presented in Fig. 4.

Target dose

All measured point doses of IM inside the PTV were less than the corresponding planned doses. The error of the measured point doses for 3D-CRT was $-2.8 \%$ and $-6.2 \%$ for R05 and R10, respectively, and those for VMAT were $-4.9 \%$ and $-8.5 \%$, respectively. The average dose error caused by respiratory movement in VMAT was larger than that of 3D-CRT by a factor of 1.4. In both 3D-CRT and VMAT, the dose error increased by a factor of 2.2 and 1.7, respectively, as the respiratory movement was doubled from R05 to R10. In the case of $D_{I M N}$, a similar trend of dose difference was observed between R05 and R10, but the difference between 3D-CRT and VMAT was negligible, as shown in Table 2. 
Table 2

Absorbed point dose values measured using GRD elements

\begin{tabular}{|c|c|c|c|c|c|c|c|c|c|c|}
\hline \multirow[t]{3}{*}{ Site } & \multicolumn{5}{|c|}{ 3D-CRT } & \multicolumn{5}{|c|}{ VMAT } \\
\hline & \multirow{2}{*}{$\begin{array}{l}\text { R00 } \\
\text { Dose }\end{array}$} & \multicolumn{2}{|l|}{ R05 } & \multicolumn{2}{|l|}{ R10 } & \multirow{2}{*}{$\begin{array}{l}\text { R00 } \\
\text { Dose }\end{array}$} & \multicolumn{2}{|l|}{ R05 } & \multicolumn{2}{|l|}{ R10 } \\
\hline & & Dose & Error & Dose & Error & & Dose & Error & Dose & Error \\
\hline \multicolumn{11}{|c|}{ GRD (point dose) } \\
\hline IM & 1.78 & 1.73 & $-2.8 \%$ & 1.67 & $-6.2 \%$ & 2.24 & 2.13 & $-4.9 \%$ & 2.05 & $-8.5 \%$ \\
\hline $\mathrm{CW}$ & 2.01 & 2.00 & $-0.5 \%$ & 1.89 & $-6.0 \%$ & 2.09 & 2.05 & $-1.9 \%$ & 1.98 & $-5.3 \%$ \\
\hline LAD & 0.10 & 0.10 & $0.0 \%$ & 0.11 & $10.0 \%$ & 0.75 & 0.57 & $-24.0 \%$ & 0.86 & $14.7 \%$ \\
\hline \multicolumn{11}{|c|}{ Film (averaged dose) } \\
\hline $\mathrm{D}_{\mathrm{IMN}}$ & 1.98 & 1.93 & $-2.5 \%$ & 1.90 & $-4.0 \%$ & 2.04 & 1.98 & $-2.9 \%$ & 1.96 & $-3.9 \%$ \\
\hline$D_{\text {lung }}$ & 1.88 & 1.82 & $-3.2 \%$ & 1.82 & $-3.2 \%$ & 0.75 & 0.75 & $0.0 \%$ & 0.76 & $1.3 \%$ \\
\hline \multicolumn{11}{|c|}{ * All dose values are expressed in Gy. } \\
\hline
\end{tabular}

All measured point doses of CW were also less than the corresponding planned doses. The error of the measured point doses for 3D-CRT was $-0.5 \%$ and $-6.0 \%$ for R05 and R10, respectively, and those for VMAT were $-1.9 \%$ and $-5.3 \%$, respectively. Under the R05 condition, the CW dose error of VMAT was much higher than that of 3D-CRT by a factor of 3.8. However, the dose error of 3D-CRT became slightly higher under the R10 condition. In both 3D-CRT and VMAT, the CW dose error significantly increased as the respiratory movement became larger, as shown in Fig. 3b.

In the gamma evaluation of measured film patches covering both IMN and CW, gamma pass rates of 3DCRT were $73.8 \%$ (R05) and 58.8\% (R10) and the gamma failing points (see red points shown in Figs. 4c and $4 \mathrm{e}$ ) tended to be evenly distributed within the beam path. The gamma failing points of VMAT were concentrated in several areas that have irregular dose distributions, as shown in Figs. $4 \mathrm{~d}$ and $4 \mathrm{f}$. The gamma pass rates of VMAT were $97.0 \%$ (R05) and $87.5 \%$ (R10). In both 3D-CRT and VMAT, gamma pass rates decreased as respiratory movements increased. The overall gamma pass rates are plotted in Fig. 5.

OAR dose

At $\mathrm{D}_{\text {lung, }}$, the dose errors of 3D-CRT were $3.2 \%$ at both R05 and R10 conditions, and VMAT showed no dose error at R05 and 1.3\% at R10. The dose error of 3D-CRT was higher than that of VMAT in both R05 and R10, as shown in Table 2. The measured dose value of 3D-CRT under the R00 condition as a reference dose was very small ( $0.1 \mathrm{~Gy}$ ) at LAD because the measured position was far from the treatment beam path; therefore, the analysis of this dose error was not significant. In the case of VMAT, 
the reference dose was $0.75 \mathrm{~Gy}$ and the dose errors were $-24.0 \%$ and $14.7 \%$ for the R05 and R10 conditions, respectively.

\section{Discussion}

Li et al. reported that the maximum point dose error due to respiration in intensity-modulated RT (IMRT) was estimated to be up to $6.2 \%$ based on RT plans, assuming a patient breathing cycle of 8 seconds (11). In our results, for IM, dose errors due to respiration in 3D-CRT were $2.8 \%$ and $6.2 \%$ for R05 and R10, respectively, while those in VMAT were slightly larger, $4.9 \%$ and $8.5 \%$, respectively. For CW, they were $0.5 \%$ and $-6.0 \%$ in 3D-CRT for R05 and R10, respectively, and those in VMAT were $-1.9 \%$ and $-5.3 \%$, respectively. Therefore, it could be assumed that the experimental design with the anthropomorphic phantom used in this study reflected the clinical situation to some extent.

Currently, elective regional nodal irradiation after mastectomy for breast cancer has been used increasingly, based on the two large randomized clinical trials. The first trail, the European Organization for Research and Treatment of Cancer 22922/10925, showed a significant reduction in breast cancer mortality and breast cancer recurrence by IMN and medial supraclavicular lymph node irradiation in stage I-III breast cancer (12). The second trial, the NCIC Clinical Trials Group MA.20 study, showed that the addition of regional nodal irradiation to the whole-breast irradiation in patients with node-positive or highrisk node-negative breast cancer reduced the recurrence rate of breast cancer (3). In a population-based cohort study from the Danish Breast Cancer Cooperative Group, RT to IMN showed an increase in overall survival in patients with early stage node-positive breast cancer (13). To take the advantage of elective nodal irradiation after mastectomy in breast cancer, precise lesion targeting and avoidance of normal tissue irradiation are important. In a systematic review of radiation dosimetry of 13,000 women in 14 trials by the Early Breast Cancer Trialists Collaborative Group, nodal RT showed reduced breast cancer recurrence, breast cancer mortality, and overall mortality. However, the benefit was only achieved when the target nodal dose was $>85 \%$ and the mean heart dose was lower than 8 Gy (14).

One way to improve the coverage of CTV is using arc-therapy techniques such as VMAT. Previous dosecomparison studies showed that arc-therapy offered improved coverage of CTV compared to that of fieldbased RT $(15,16)$. However, in case of postmastectomy radiation, it has not been proven whether the benefit of VMAT would be achieved because the width of CW after mastectomy may be very narrow and respiratory movement existed. In our study, for example, the mean dose error of both IM and CW increased by a factor of 7.1 and 2.3 in 3D-CRT and VMAT, respectively, as the respiratory amplitude was doubled. However, the absolute value of the dose error of VMAT was higher than that of 3D-CRT in all cases. In addition, the dose errors of both 3D-CRT and VMAT were less than $5 \%$ under the shallow breathing of R05.

Meanwhile, from the viewpoint of overall dose errors due to breathing, dose distributions of VMAT were more accurate and robust than those of 3D-CRT in both R05 and R10, as shown in Fig. 4. A relatively high gamma passing rate of VMAT may be expected, since the VMAT could paint the prescribed dose evenly 
on the target while suppressing the absorbed dose to the surroundings by precise beam modulation, whereas the dose distribution of 3D-CRT was only dependent on the beam characteristics of the percent depth dose. However, it should be noted that dose errors could be concentrated on the spot, such as nodal lesions where the beam modulation is highly applied, as shown in Fig. $4 \mathrm{f}$, when the respiratory movement is enlarged. Therefore, it is important to keep the patient's breathing shallow during VMAT.

Pedersen et al. reviewed $16 \mathrm{CT}$ scans of adjuvant RT plans for breast cancer and found that the mean chest wall excursion at the position of the xiphoid process was $2.5 \mathrm{~mm}$ (range: 1-4 mm) during freebreathing (17). Lowanichkiattikul et al. also reported the respiratory movement of $\mathrm{CW}$ using data from 38 patients. They placed radio-opaque markers at four anatomical landmark points and checked the width of maximal $\mathrm{CW}$ movement. Although the respiratory movement of $\mathrm{CW}$ in the anterior-posterior direction was the largest among the three directions in general, the movement only ranged from 4.2. to $5.4 \mathrm{~mm}$ at all points (18). Thus, we expected that the R05 condition would be generally achievable by patients and that VMAT could be implemented with sufficient accuracy.

The $\mathrm{D}_{\text {lung }}$ value of VMAT was only $39.9 \%$ of that of 3D-CRT, as expected, and we also noted that the difference in dose errors between R05 and R10 was negligible in both 3D-CRT and VMAT, as shown in Fig. 6. In VMAT, the effect of respiratory movement on the surrounding doses seems to be limited because VMAT intends to suppress the surrounding dose. However, in the case of 3D-CRT, various planning conditions such as beam geometry and patient anatomy should also be examined.

For $L A D$, all measured dose values were very low because the point was far from the PTV; hence, the dose errors caused by respiratory movement were not significant. However, we noted that the measured LAD dose of VMAT was higher than that of 3D-CRT by a factor of 7.5. Therefore, in spite of the prominent target coverage of VMAT, planned doses of organs at risk should be carefully monitored (16). According to a systematic review of heart doses for breast RTs published between 2003 and 2013, the mean heart dose was 4.2 Gy when no IMN was included, but it increased to 8 Gy with IMN (19).

For sparing the heart and eliminating the effect of respiratory movement during left breast RT, the deep inspiration breath-hold (DIBH) technique could be combined with VMAT. Nissen et al. reported that the mean heart dose was reduced by $48 \%$ (from 5.2 Gy to $2.7 \mathrm{~Gy}$ ) using both DIBH and IMRT (20). Hayden et al. also reported that the DIBH showed a significant reduction in heart and LAD doses in IMRT compared to free breathing technique (21). Although the DIBH is optimal to avoid the dose error caused by respiratory movement, it could not be available in a variety of situations, such as long treatment time or in certain patient's clinical conditions. The overall dosimetric impact caused by respiratory movement seemed to be relatively small in VMAT according to gamma evaluation results, as shown in Fig. 4 . The gamma pass rate of VMAT under the R10 condition was even higher than that of 3D-CRT under the R05 condition. In particular, the gamma pass rate of VMAT reached $97.0 \%$ under R05 conditions. Therefore, we expect that the sufficient accuracy of VMAT could be maintained if the patient maintained shallow breathing during the treatment. Nevertheless, it may be necessary to evaluate PTV coverage in the case of complex target shapes owing to the concerns about possible underdose delivery. 
This study has three limitations that come from our phantom-based experimental design. First, although the exact respiratory movement should be simulated using three-dimensional movements, we ignored the medial-lateral (M-L) movement due to the limitation of our experimental apparatus. It was also considered that the $M-L$ movement was the smallest among the three-dimensional breathing motions (22). Second, although the anatomical geometry of the chest wall may be highly dependent on the individual patient, the analysis of dose errors implemented in this study was only based on the given anthropomorphic phantom. Nevertheless, we expected that our study could provide an overview of the dosimetric impact of respiratory movement on 3D-CRT and VMAT techniques, respectively. Third, we evaluated the relationship between respiratory movement and the corresponding dose errors measured from single fractionation. The dose errors arising from all the fractionations could be partially summed up or canceled out. The quality assurance of dose per fractionation is still important.

\section{Conclusions}

If the patient maintained shallow to normal breathing, PMRT including IMNs could be implemented with acceptable accuracy. In particular, it was possible to maintain the advantages of VMAT, which enabled high-target coverage and OAR protection.

\section{Declarations}

\section{Ethics approval and consent to participate:}

Not applicable because this article does not contain any studies with human or animal subjects.

\section{Consent for publication:}

Not applicable

\section{Availability of data and materials:}

Not applicable

\section{Competing interests:}

The authors declare that they have no competing interests.

\section{Funding:}

This study was supported by the Research Institute for Convergence of Biomedical Science and Technology Grant (30-2019-013), Pusan National University Yangsan Hospital. 


\section{Authors' contributions:}

YK and WK participated in the study design. HJ and DWK performed the experiments and analyses. JN, DHK, and DP interpreted the data. HJ and JHJ drafted the manuscript, which was reviewed and approved in its final form by the other authors.

\section{Acknowledgements:}

Not applicable.

\section{References}

1. Ebctcg McGaleP, Taylor C, Correa C, Cutter D, Duane F, et al. Effect of radiotherapy after mastectomy and axillary surgery on 10-year recurrence and 20-year breast cancer mortality: meta-analysis of individual patient data for 8135 women in 22 randomised trials. Lancet. 2014;383(9935):2127-35.

2. Van de Steene J, Soete G, Storme G. Adjuvant radiotherapy for breast cancer significantly improves overall survival: the missing link. Radiother Oncol. 2000;55(3):263-72.

3. Whelan TJ, Olivotto IA, Parulekar WR, Ackerman I, Chua BH, Nabid A, et al. Regional Nodal Irradiation in Early-Stage Breast Cancer. N Engl J Med. 2015;373(4):307-16.

4. Frasier LL, Holden S, Holden T, Schumacher JR, Leverson G, Anderson B, et al. Temporal trends in postmastectomy radiation therapy and breast reconstruction associated with changes in National Comprehensive Cancer Network Guidelines. JAMA Oncol. 2016;2(1):95-101.

5. Clarke M, Collins R, Darby S, Davies C, Elphinstone P, Evans V, et al. Effects of radiotherapy and of differences in the extent of surgery for early breast cancer on local recurrence and 15-year survival: an overview of the randomised trials. Lancet. 2005;366(9503):2087-106.

6. Darby SC, Ewertz M, McGale P, Bennet AM, Blom-Goldman U, Bronnum D, et al. Risk of ischemic heart disease in women after radiotherapy for breast cancer. The New Eng J Med. 2013;368(11):987-98.

7. Otto K. Volumetric modulated arc therapy: IMRT in a single gantry arc. Med Phys. 2008;35(1):310-7.

8. Yu CX, Tang G. Intensity-modulated arc therapy: principles, technologies and clinical implementation. Phys Med Biol. 2011;56(5):R31-54.

9. Popescu CC, Olivotto IA, Beckham WA, Ansbacher W, Zavgorodni S, Shaffer R, et al. Volumetric modulated arc therapy improves dosimetry and reduces treatment time compared to conventional intensity-modulated radiotherapy for locoregional radiotherapy of left-sided breast cancer and internal mammary nodes. Int J Radiat Oncol Biol Phys. 2010;76(1):287-95.

10. Low DA, Harms WB, Mutic S, Purdy JA. A technique for the quantitative evaluation of dose distributions. Med Phys. 1998;25(5):656-61.

11. Li H, Park P, Liu W, Matney J, Liao Z, Balter P, et al. Patient-specific quantification of respiratory motion-induced dose uncertainty for step-and-shoot IMRT of lung cancer. Med Phys. 
2013;40(12):121712.

12. Poortmans PM, Collette S, Kirkove C, Van Limbergen E, Budach V, Struikmans $H$, et al. Internal Mammary and Medial Supraclavicular Irradiation in Breast Cancer. The New Eng Med. 2015;373(4):317-27.

13. Thorsen LB, Offersen BV, Dano H, Berg M, Jensen I, Pedersen AN, et al. DBCG-IMN: A PopulationBased Cohort Study on the Effect of Internal Mammary Node Irradiation in Early Node-Positive Breast Cancer. J Clin Oncol. 2016;34(4):314-20.

14. Dodwell D, Taylor C, McGale P, Coles C, Duane F, Gray R, et al. Abstract GS4-02: Regional lymph node irradiation in early stage breast cancer: An EBCTCG meta-analysis of 13,000 women in 14 trials. 2019;79(4 Supplement):GS4-02-GS4-.

15. Ranger A, Dunlop A, Hutchinson K, Convery H, Maclennan MK, Chantler $\mathrm{H}$, et al. A Dosimetric Comparison of Breast Radiotherapy Techniques to Treat Locoregional Lymph Nodes Including the Internal Mammary Chain. Clin Oncol. 2018;30(6):346-53.

16. Lomax AJ, Cella L, Weber D, Kurtz JM, Miralbell R. Potential role of intensity-modulated photons and protons in the treatment of the breast and regional nodes. Int $\mathrm{J}$ Radiat Oncol Biol Phys. 2003;55(3):785-92.

17. Pedersen AN, Korreman S, Nystrom H, Specht L. Breathing adapted radiotherapy of breast cancer: reduction of cardiac and pulmonary doses using voluntary inspiration breath-hold. Radiother Oncol. 2004;72(1):53-60.

18. Lowanichkiattikul C, Dhanachai M, Sitathanee C, Khachonkham S, Khaothong P. Impact of chest wall motion caused by respiration in adjuvant radiotherapy for postoperative breast cancer patients. SpringerPlus. 2016;5:144.

19. Taylor CW, Wang Z, Macaulay E, Jagsi R, Duane F, Darby SC. Exposure of the Heart in Breast Cancer Radiation Therapy: A Systematic Review of Heart Doses Published During 2003 to 2013. Int J Radiat Oncol Biol Phys. 2015;93(4):845-53.

20. Nissen HD, Appelt AL. Improved heart, lung and target dose with deep inspiration breath hold in a large clinical series of breast cancer patients. Radiother Oncol. 2013;106(1):28-32.

21. Hayden AJ, Rains M, Tiver K. Deep inspiration breath hold technique reduces heart dose from radiotherapy for left-sided breast cancer. J Med Imaging Radiat Oncol. 2012;56(4):464-72.

22. Pettersson N, Simpson D, Atwood T, Hattangadi-Gluth J, Murphy J, Cervino L. Automatic patient positioning and gating window settings in respiratory-gated stereotactic body radiation therapy for pancreatic cancer using fluoroscopic imaging. J Appl Clin Med Phys. 2018;19(2):74-82.

\section{Abbreviations}

PMRT post-mastectomy radiation therapy

IMN internal mammary node 
VMAT volumetric modulated arc therapy

3D-CRT three-dimensional conformal radiation therapy

CW chest wall

$L A D$, left anterior descending

RT Radiation therapy

PTV planning target volume

RTP radiation treatment plans

OAR organ at risk

GRD glass rod dosimeters

IMRT intensity-modulated RT

DIBH deep inspiration breath-hold

M-L medial-lateral

\section{Figures}

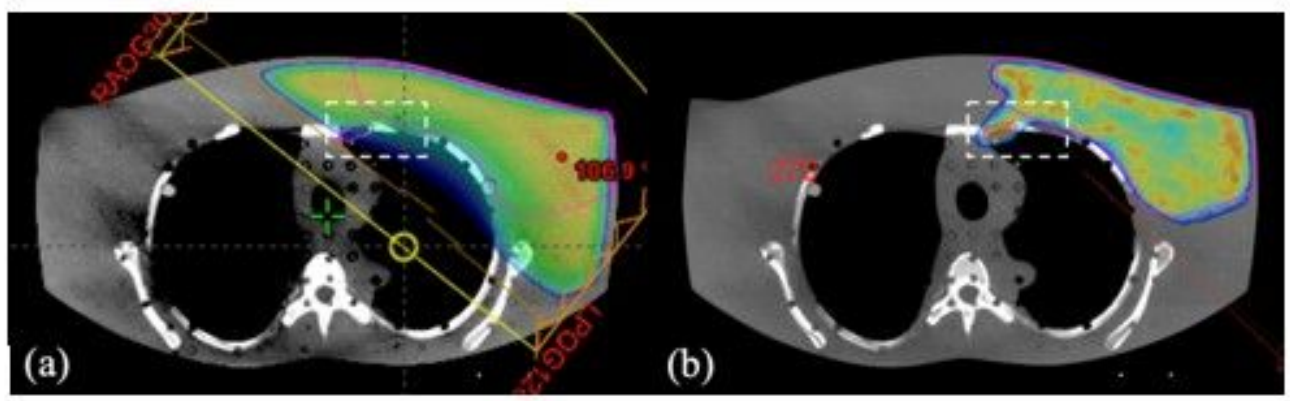

Figure 1.

Figure 1 
Field geometry of (a) 3D-CRT and (b) VMAT plans. The film patches were laid on a white dotted area for the measurement of dose distribution. 3D-CRT, three-dimensional conformal radiation therapy; VMAT, volumetric modulated arc therapy

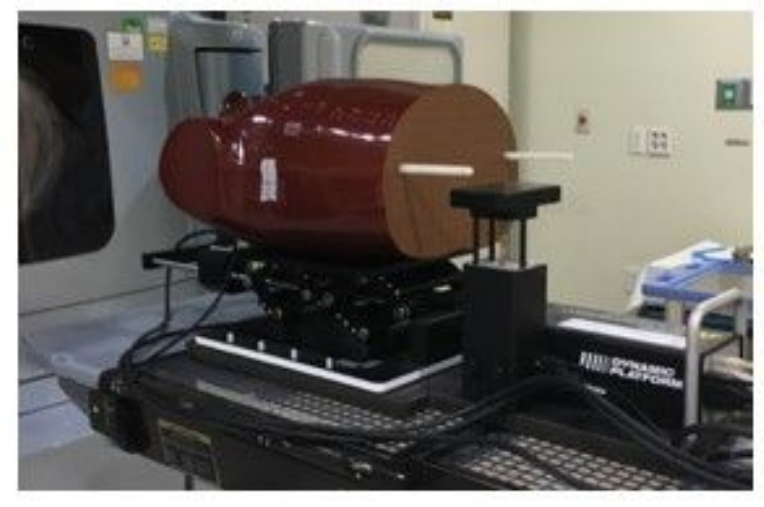

\section{Figure 2}

The experimental setup of the two-dimensional dynamic platform

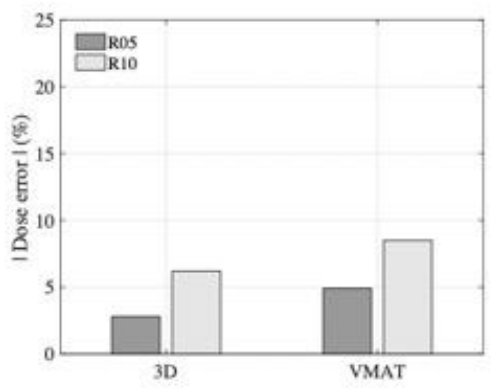

(a) IMI

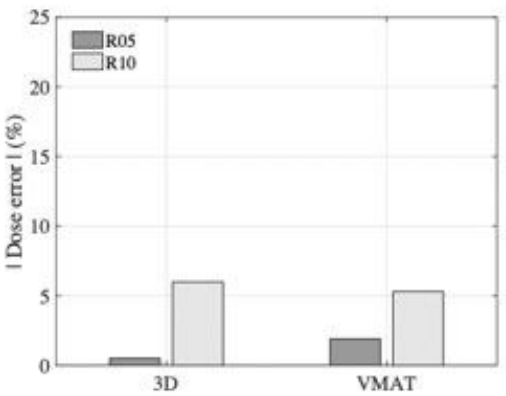

(b) $\mathrm{CW}$

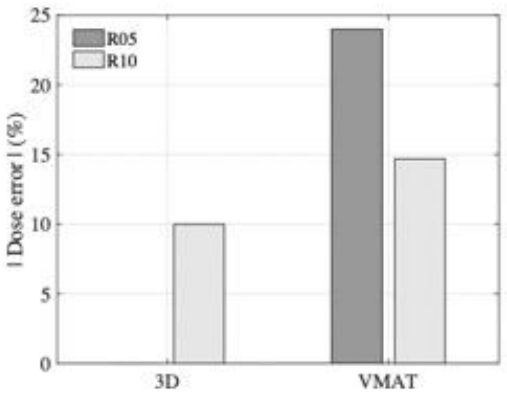

(c) LAD

Figure 3 
Absorbed dose errors with respect to the respiration amplitude and dose delivery techniques in (a) internal mammary nodes, (b) chest wall, and (c) left anterior descending artery. All errors are presented as absolute values.
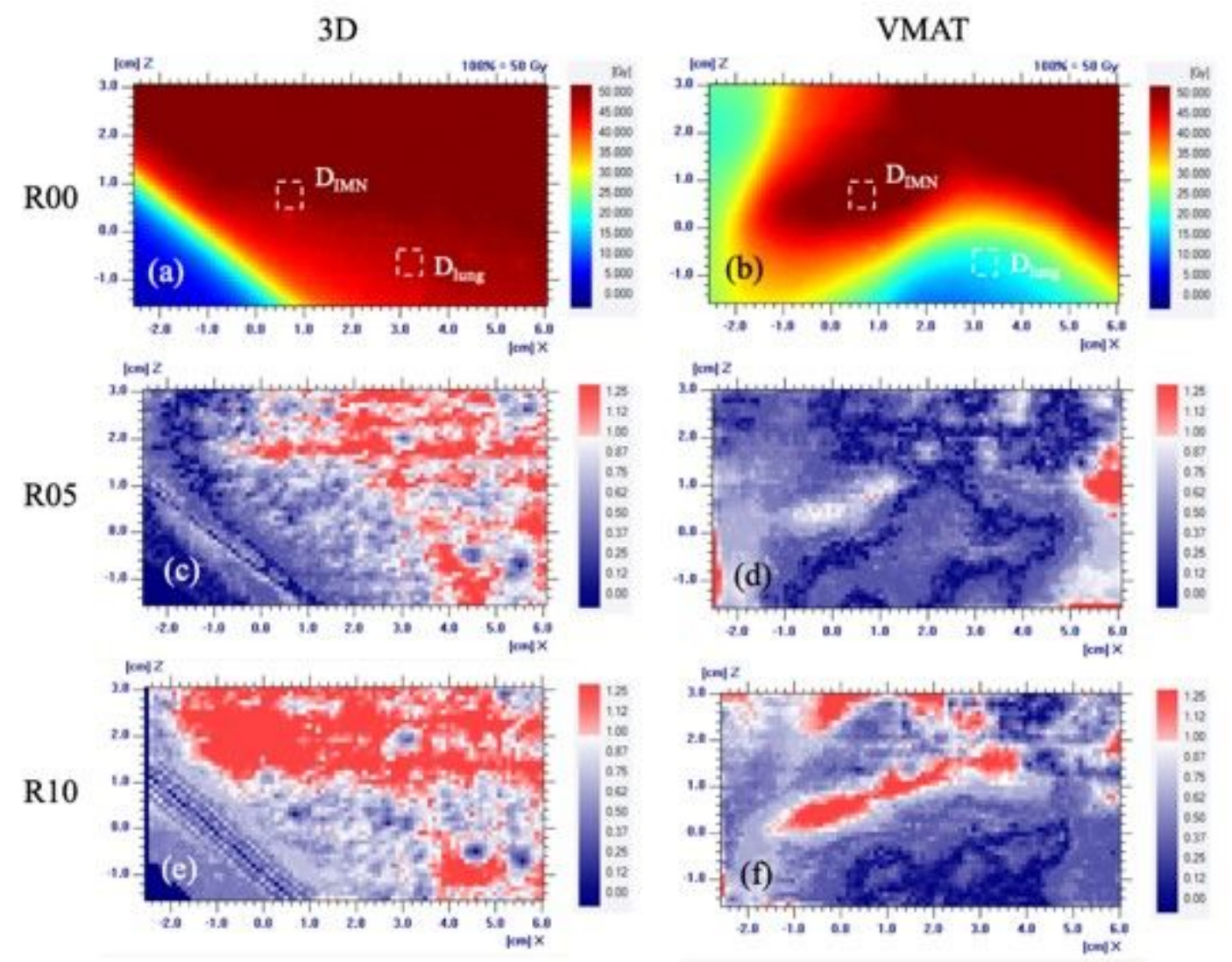

\section{Figure 4}

Measured dose distributions (multiplied by 25 fractionations) using film patches (a) 3D-CRT and (b) VMAT under R00 condition, and gamma evaluation results of 3D-CRT (c, e) and VMAT (d, f) under R05 and R10 conditions, respectively. DIMN and Dlung (dotted white squares) indicate the location of the IMN (inside the PTV) and lung (outside the PTV), respectively. 


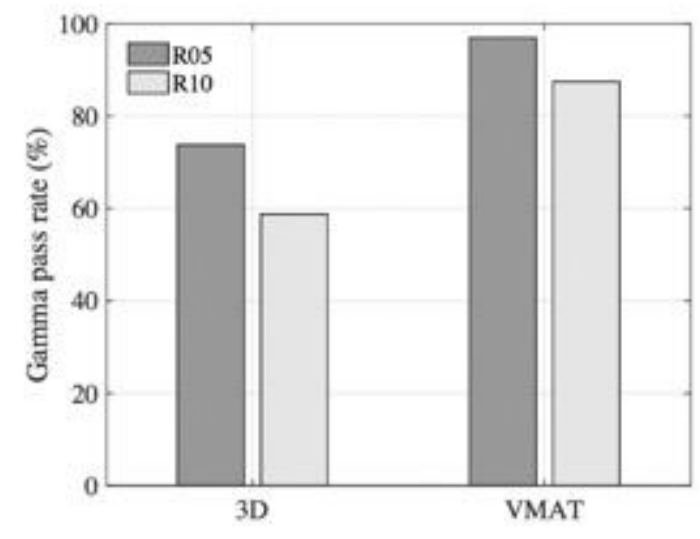

Figure 5

Gamma passing rates with respect of respiratory movements and treatment techniques

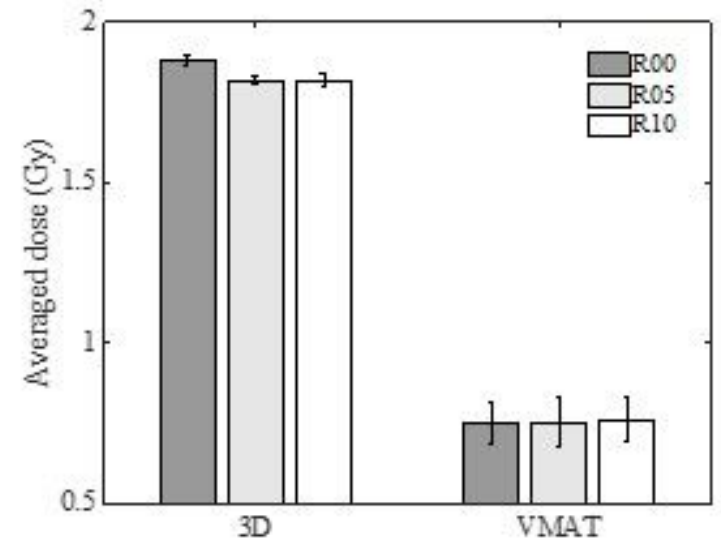

Figure 6

Comparison of the averaged dose values between 3D-CRT and VMAT in the lungs 